\title{
Efficient supercapacitor energy usage in mobile phones
}

\author{
José Monteiro \\ Instituto Universitário de Lisboa (ISCTE-IUL) \\ Lisboa, Portugal \\ jjnmonteiro@gmail.com
}

\author{
Nuno Garrido \\ Instituto Universitário de Lisboa (ISCTE-IUL), \\ Instituto de Telecomunicações (IT-IUL) \\ Lisboa, Portugal \\ nuno.garrido@iscte.pt
}

\author{
Ricardo Fonseca \\ Instituto Universitário de Lisboa (ISCTE-IUL) \\ GoLP / Instituto de Plasmas e Fusão Nuclear \\ Lisboa, Portugal \\ ricardo.fonseca@iscte.pt
}

\begin{abstract}
This paper proposes a solution for using supercapacitors to power mobile phones. Based on a DC/DC voltage converter and a supercapacitor stacking scheme, this solution is designed to maximize the usable energy of the supercapacitors as well as the converter's performance, which is confirmed in simulation. A specific charging scheme for this design is also proposed and simulated, enabling fast and efficient charging of the mobile device.
\end{abstract}

Keywords - supercapacitors; mobile phones, energy efficiency, fast charging

\section{INTRODUCTION}

Mobile electronic devices are ubiquitous in contemporary society. Their autonomy relies on efficient storage of electrical energy, generally through the use of electro-chemical batteries, of which Li-ion batteries have currently the largest widespread use. However, while providing the required energy density and autonomy, these batteries exhibit lengthy and inefficient charging cycles, requiring several hours to attain the required charge, whilst having a short lifespan.

An alternative technology for this purpose is the use of electric double-layer capacitors (EDLCs), also known as supercapacitors. Supercapacitors are high power density, long life, electrical energy storage devices, that exhibit electrical properties, namely charging capability, similar to those of conventional capacitors, and therefore promise an order of magnitude shorter charging cycles comparing with those of conventional batteries [1]-[2]. At the present time, the biggest disadvantage of supercapacitors is that they have relatively low energy density when compared with batteries, although this is expected to change in the near future [3]-[4]. Considering all its advantages and disadvantages when compared with Li-ion batteries [2], this paper proposes the use of supercapacitors in mobile devices in order to achieve faster and more efficient charges.
The main technical difficulty lies in matching the supercapacitor cell voltage with the constant $3.7 \mathrm{~V}$ required by the electronic device. This paper proposes a solution, combining the use of a $\mathrm{DC} / \mathrm{DC}$ voltage converter with a supercapacitor stacking scheme in order to solve the non-fixed over time, low supercapacitor cell voltage problem, ensuring that most of the stored energy is usable, whilst taking full advantage of the DC/DC converter's efficiency. Theoretical limits are derived and simulation results are presented, showing an effective autonomy gain by using the stacking approach versus the use of a DC/DC converter only. The influence of some non-idealities of this scheme are quantified and analyzed, namely the sheet-resistance of the switches. Finally, an application specific charging method is proposed, enabling fast, parallel charge of the supercapacitors.

\section{USING SUPERCAPACITORS TO POWER MOBILE PHONES}

A mobile phone is usually designed to operate with an input voltage of $3.7 \mathrm{~V}$. With this requirement come two problems of using supercapacitors for this purpose: i) low maximum voltage per cell, typically $2.7 \mathrm{~V}$ (below the required $3.7 \mathrm{~V}$ ) and ii) non-constant voltage across the supercapacitor as it discharges $(V=Q / C)$. Two simple solutions can be used to solve these problems: i) stacking, at least, two supercapacitor cells to get a higher voltage and use a step-down (buck) converter to fix the voltage or ii) using a single cell with a step-up (boost) converter to boost and fix the voltage.

To estimate the usable energy in the supercapacitor we will focus on solution ii), given that solution i) requires complex circuitry to balance the charge across each supercapacitor [5] of the stack. Letting $V_{\mathrm{C}(\mathrm{MAX})}$ be the maximum voltage across a supercapacitor and $V_{\mathrm{IN}(\mathrm{MIN})}$ the minimum input voltage for the step-up converter to operate, the usable energy $U E$ of the supercapacitor is given by (1). 


$$
U E=E_{\mathrm{HARNESSED}} / E_{\mathrm{TOTAL}}=1-\left(V_{\mathrm{IN}(\mathrm{MIN})} / V_{\mathrm{C}(\mathrm{MAX})}\right)^{2}
$$

Typically, low input voltage boost converters work with input voltages down to $1.0 \mathrm{~V}$, which leads to the usable energy being approximately $86 \%$ of the total energy stored in the supercapacitor. While this value would be acceptable for some applications, we further need to account for the energy losses in the step-up converter. Step-up converters are not ideal, and their efficiency commonly decreases with the augment of the difference between output and input voltages, which is unavoidable when using supercapacitors at the input.

Therefore, to minimize the inaccessible energy from the supercapacitor and to allow the converter to operate in a higher efficiency input voltage range, this paper proposes the use of a voltage controlled supercapacitor switching module combined with a boost converter, as shown in Fig. 1.

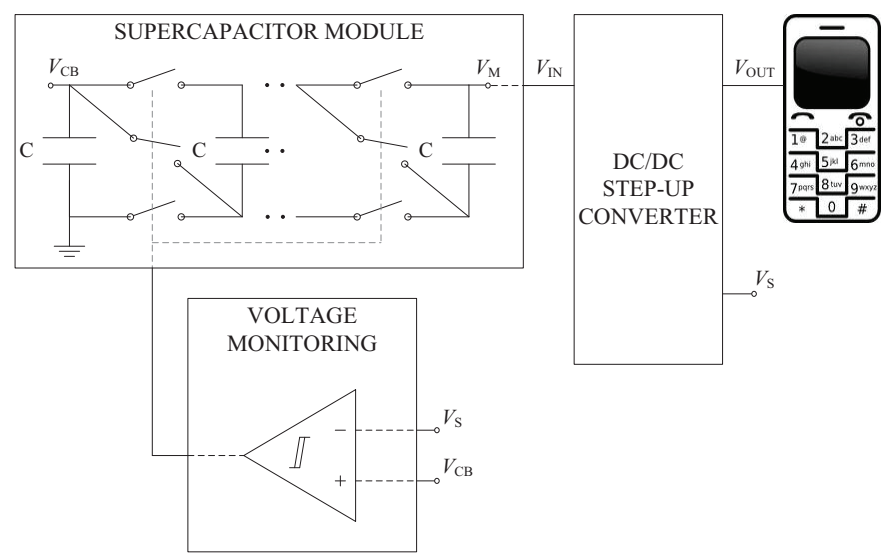

Figure 1. Voltage monitoring and supercapacitor switching module

This scheme enables $V_{\mathrm{M}}$ (the input for the converter) to double, by stacking half of the supercapacitors on top of the other half, when its value drops below a certain voltage $V_{\mathrm{S}}$ (switching voltage). The switching is controlled by Schmitt triggered comparators (in the voltage monitoring block) which get the switching voltage reference $V_{\mathrm{S}}$ from the step-up converter fixed output voltage. For a set of $2^{N}$ capacitors, this procedure can be done $N$ times, leading to a usable energy value given by:

$$
U E=1-\left[\left(V_{\mathrm{IN}(\mathrm{MIN})} / 2^{N}\right) / V_{\mathrm{C}(\mathrm{MAX})}\right]^{2}
$$

Since this value is not directly dependent on the switching voltage, $V_{\mathrm{S}}$ can be chosen according to the highest efficiency input range of the step-up converter that is used. Table I shows the effect of $N$ in the value of the usable energy, along with the complexity added to the system. The number of switches, presented in this table, assumes that they are single-pole single-throw (SPST) type. Instead, if single-pole double-throw (SPDT) switches are used, the number of switches is reduced by $1 / 3$. Also, if SPSTs are used, there is a need for additional circuitry to logically invert the control signal for the switches, once some are closed while others are opened without overlapping.
Table I. Effect of $N$ in the usable energy and system complexity

\begin{tabular}{|c|c|c|c|c|c|}
\hline \# switchings $(\boldsymbol{N})$ & $\mathbf{0}$ & $\mathbf{1}$ & $\mathbf{2}$ & $\mathbf{3}$ & $\mathbf{4}$ \\
\hline Usable Energy $(\boldsymbol{U E})^{\mathbf{a}}$ & $86.3 \%$ & $96.6 \%$ & $99.1 \%$ & $99.8 \%$ & $99.9 \%$ \\
\hline \# supercaps & 1 & 2 & 4 & 8 & 16 \\
\hline \# voltage comparators & 0 & 1 & 2 & 3 & 4 \\
\hline \# switches & 0 & 3 & 9 & 21 & 45 \\
\hline
\end{tabular}

Table II shows the theoretical limits for the usable energy given by (2), for different values of $V_{\mathrm{IN}(\mathrm{MIN})}$ and $N$, showing that $N=3$ is clearly a limit for this scheme when using a boost converter with an operating input voltage within the considered range (from $0.7 \mathrm{~V}$ to $1.7 \mathrm{~V}$ ). Nevertheless, the practical choice of the number of stacking operations $(N)$ should be done in accordance with the converter's characteristics, namely the minimum operating input voltage, and with the desired complexity for the application where it is used.

The values presented in Table II the maximum supercapacitor cell voltage $V_{\mathrm{C}(\mathrm{MAX})}=2.7 \mathrm{~V}$, the typical value for EDLCs, correspond to the worst case for this scheme. Therefore, if the system is using lower voltage supercapacitors, like several hybridcapacitors and pseudocapacitors, the augment in the relative gains observed in Table II, with the increase of $V_{\mathrm{IN}(\mathrm{MIN})}$, would be even greater.

Table II. Impact of $N$ and $V_{\mathrm{IN}(\mathrm{MIN})}$ in the usable energy $\left(V_{\mathrm{C}(\mathrm{MAX})}=2.7 \mathrm{~V}\right)$

\begin{tabular}{|c|c|c|c|c|c|}
\hline \multicolumn{2}{|c|}{ \# switchings $(N)$} & 0 & 1 & 2 & 3 \\
\hline \multirow{4}{*}{} & $V_{I N(M I N)}=0.7 V$ & $93.3 \%$ & $98.3 \%$ & $99.6 \%$ & $99.9 \%$ \\
\cline { 2 - 6 } & $V_{I N(M I N)}=\mathbf{0 . 9} \boldsymbol{V}$ & $88.9 \%$ & $97.2 \%$ & $99.3 \%$ & $99.8 \%$ \\
\cline { 2 - 6 } & $V_{I N(M I N)}=1.1 V$ & $83.4 \%$ & $95.9 \%$ & $99.0 \%$ & $99.7 \%$ \\
\cline { 2 - 6 } & $V_{I N(M I N)}=1.3 V$ & $76.8 \%$ & $94.2 \%$ & $98.6 \%$ & $99.6 \%$ \\
\cline { 2 - 6 } & $V_{I N(M I N)}=1.5 V$ & $69.1 \%$ & $92.3 \%$ & $98.1 \%$ & $99.5 \%$ \\
\cline { 2 - 6 } & $V_{I N(M I N)}=1.7 V$ & $60.4 \%$ & $90.1 \%$ & $97.5 \%$ & $99.4 \%$ \\
\hline
\end{tabular}

\section{SimUlation RESUlTS}

The proposed circuit, depicted in Fig. 1, was simulated using a model of Linear Technology's LTC3401, which is a DC/DC step-up converter that operates with input voltages below $1.0 \mathrm{~V}$, and the LTC1841 for the voltage comparators, since this is an ultralow power comparator, minimizing its impact on the overall consumption. The supercapacitor module was designed for a total capacitance of $100 \mathrm{~F}$ composed of four $25 \mathrm{~F}$ supercapacitors (either for $N=0,1$ or 2 ), with an initial voltage of $2.7 \mathrm{~V}$ across each cell, making a total energy of $101.25 \mathrm{mWh}$ (approximately $1 / 30$ of the maximum stored energy of a typical Li-ion battery). The supercapacitors leakage currents are not considered at this point. To emulate the mobile phone consumption, the output voltage of the step-up converter was fixed at $3.7 \mathrm{~V}$ and the output load was set to consume about $200 \mathrm{~mA}$ during call mode and $6.4 \mathrm{~mA}$ in standby mode, as these are the average measured current consumptions of a Nokia 3310 , at $3.7 \mathrm{~V}$. We first considered ideal SPST switches $\left(R_{\mathrm{ON}}=0 \Omega\right)$. 
Fig. 2 illustrates the typical behavior of the input (supercapacitor module) and output (to the mobile phone) voltages of the step-up converter for $N=2$ in standby mode. The output from the boost converter shows minimum behavior changes when the commutations occur.

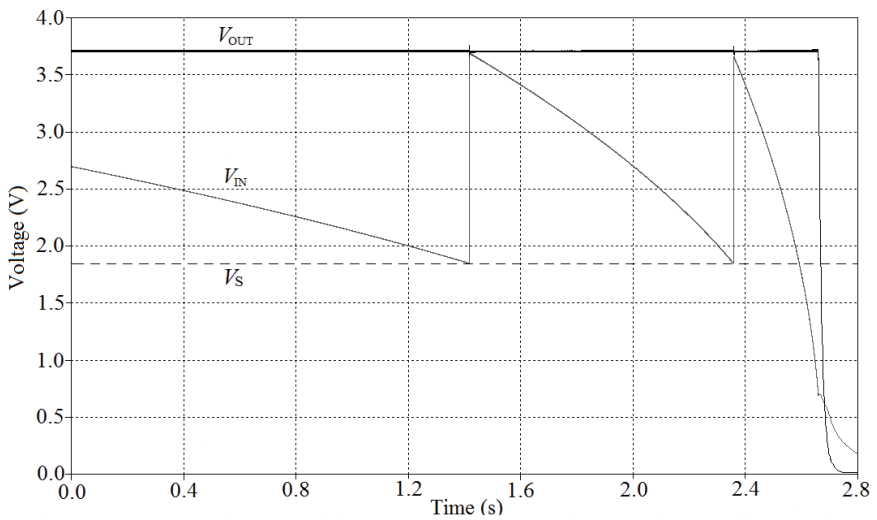

Figure 2. $\quad V_{\mathrm{IN}}$ and $V_{\text {OUT }}$ of the step-up converter for $N=2$ in standby mode

The overall results of these simulations ${ }^{1)}$ are summarized in Table III and Table IV. These results show improvements in the autonomy of the mobile phone both in standby and call modes, being more significant in call mode. This happens since the boost converter's minimum operating input voltage $V_{\mathrm{IN}(\mathrm{MIN})}$ in call mode (high output current) is higher than in standby mode (low output current), resulting in superior relative gains for the energy harnessed for each stacking operation, as shown by (2). Moreover, the boost efficiency is improved in call mode, as a result of the increase of its average input voltage.

Table III. Simulation results for standby mode

\begin{tabular}{|c|c|c|c|c|c|c|c|}
\hline \multirow{2}{*}{$N$} & \multirow{2}{*}{$\begin{array}{c}\text { Avg. } \\
V_{\text {IN }}(\mathrm{V})\end{array}$} & \multirow{2}{*}{$\begin{array}{c}V_{\mathrm{IN}(\mathrm{MIN})}{ }^{\mathrm{a}} \\
(\mathrm{V})\end{array}$} & \multirow{2}{*}{$\begin{array}{c}\text { Boost } \\
\text { efficiency }^{\mathrm{a}}\end{array}$} & \multicolumn{2}{|c|}{$\begin{array}{c}\text { Energy } \\
\text { harnessed }\end{array}$} & \multicolumn{2}{|c|}{ Autonomy } \\
\hline & & & & $(m W h)$ & $\operatorname{Gain}^{\mathrm{b}}$ & (h) & Gain $^{b}$ \\
\hline $\mathbf{0}$ & 1.89 & \multirow{3}{*}{0.70} & $87.7 \%$ & 94.9 & $\mathrm{n} / \mathrm{a}$ & 3.47 & $\mathrm{n} / \mathrm{a}$ \\
\hline 1 & 2.41 & & $88.0 \%$ & 99.4 & $4.8 \%$ & 3.66 & $5.3 \%$ \\
\hline 2 & 2.52 & & $88.0 \%$ & 100.4 & $5.8 \%$ & 3.69 & $6.4 \%$ \\
\hline
\end{tabular}

Table IV. Simulation results for call mode

\begin{tabular}{|c|c|c|c|c|c|c|c|}
\hline \multirow{2}{*}{$N$} & \multirow{2}{*}{$\begin{array}{l}\text { Avg. } \\
V_{\text {IN }}(V)\end{array}$} & \multirow{2}{*}{$\begin{array}{c}V_{\mathrm{IN}(\mathrm{MIN})}{ }^{\mathrm{a}} \\
\text { (V) }\end{array}$} & \multirow{2}{*}{$\begin{array}{c}\text { Boost } \\
\text { efficiency }\end{array}$} & \multicolumn{2}{|c|}{$\begin{array}{c}\text { Energy } \\
\text { harnessed }\end{array}$} & \multicolumn{2}{|c|}{ Autonomy } \\
\hline & & & & $(m W h)$ & Gain $^{\mathrm{b}}$ & (min) & Gain $^{\mathrm{b}}$ \\
\hline 0 & 1.94 & \multirow{3}{*}{0.78} & $93.1 \%$ & 92.2 & $\mathrm{n} / \mathrm{a}$ & 6.88 & $\mathrm{n} / \mathrm{a}$ \\
\hline 1 & 2.44 & & $95.9 \%$ & 98.2 & $6.5 \%$ & 7.52 & $9.3 \%$ \\
\hline 2 & 2.55 & & $96.6 \%$ & 99.6 & $8.0 \%$ & 7.68 & $11.6 \%$ \\
\hline
\end{tabular}

b. All gains are relative to values for $N=0$

\section{IMPACT OF THE SWITCH'S ON-RESISTANCE IN THE SYSTEM PERFORMANCE}

Since the proposed scheme is based on the use of switches (SPSTs or SPDTs) for stacking and unstacking the supercapacitors in the module, it's important to quantify and analyze the impact of their on-resistance $R_{\mathrm{ON}}$ in the scheme's performance, namely its effect on the supercapacitor module

\footnotetext{
1) Due to memory limitations, the total capacitance used in simulations for the supercapacitor module, was scaled down 5000 times for standby mode and 200 times for call mode
}

voltage $V_{\mathrm{M}}$ (input for the boost converter) and, consequently, on the autonomy gains.

Assuming that each supercapacitor cell in the module is fully charged at $V_{\mathrm{C}(\mathrm{MAX})}$ (i.e. all the cells are connected in parallel) and the converter is consuming a current $I_{\text {BOOST }}$ at the input, the voltage $V_{\mathrm{M}}$ seen from the converter's input is given by (3) for $N=1$ and (4) for $N=2$.

$$
\begin{aligned}
& V_{\mathrm{M}}=V_{\mathrm{C}(\mathrm{MAX})}-0.5 R_{\mathrm{ON}} I_{\mathrm{BOOST}} \\
& V_{\mathrm{M}}=V_{\mathrm{C}(\mathrm{MAX})}-1.5 R_{\mathrm{ON}} I_{\mathrm{BOOST}}
\end{aligned}
$$

When the first stacking operation occurs and half the supercapacitors are stacked on top of the other half, assuming that all the cells have the same voltage $V_{\mathrm{C}(1 \mathrm{SW})}$, the supercapacitor module voltage is given by (5) for $N=1$ and (6) for $N=2$.

$$
\begin{gathered}
V_{\mathrm{M}}=2 V_{\mathrm{C}(1 \mathrm{SW})}-R_{\mathrm{ON}} I_{\mathrm{BOOST}} \\
V_{\mathrm{M}}=2 V_{\mathrm{C}(1 \mathrm{SW})}-2 R_{\mathrm{ON}} I_{\mathrm{BOOST}}
\end{gathered}
$$

Finally, when the second stacking operation occurs, which is only possible in a module with $N=2$, and each cell has a voltage $V_{\mathrm{C}(2 \mathrm{SW})}$, the supercapacitor module voltage is given by (7).

$$
V_{\mathrm{M}}=4 V_{\mathrm{C}(2 \mathrm{SW})}-3 R_{\mathrm{ON}} I_{\mathrm{BOOST}}
$$

As expected, the voltage drop caused by the switches on-resistance will lower the effective voltage of the supercapacitor module, being this effect worse when $N=2$. Table $\mathrm{V}$ shows simulation results for the impact of the on-resistance $R_{\mathrm{ON}}$ in autonomy gains in standby and call modes.

In standby mode, a higher value for the switch on-resistance doesn't have a significant impact on the scheme's autonomy gains, since the current that is feeding the converter is relatively low, compensating higher on-resistance values. For call mode, since more current is being drawn to the boost converter, the on-resistance is responsible for greater power losses than in standby mode. This is clear for the situation of $R_{\mathrm{ON}}=R$, where the scheme's autonomy for $N=2$ is reduced to the point that its autonomy gain $(6.3 \%)$ is lower than the obtained for $N=1(6.9 \%)$. Simulations confirm that a system

Table V. Simulation results showing the effect of $R_{\mathrm{ON}}$ in the presented scheme's autonomy gains

\begin{tabular}{|c|c|c|c|c|c|c|c|c|}
\hline \multicolumn{2}{|c|}{$\boldsymbol{R}_{\mathrm{ON}}$} & $\boldsymbol{R}^{\mathrm{a}}$ & $\boldsymbol{R} / \mathbf{2}$ & $\boldsymbol{R} / \mathbf{4}$ & $\boldsymbol{R} / \mathbf{8}$ & $\boldsymbol{R} / \mathbf{1 6}$ & $\boldsymbol{R} / \mathbf{3 2}$ & $\mathbf{0}$ \\
\hline \multirow{3}{*}{ Standby } & $\boldsymbol{N}=\mathbf{1}$ & $5.2 \%$ & - & - & - & - & - & $5.2 \%$ \\
\cline { 2 - 9 } & $\boldsymbol{N}=\mathbf{2}$ & $6.2 \%$ & - & - & - & - & - & $6.3 \%$ \\
\hline \multirow{3}{*}{ Call } & $\boldsymbol{N}=\mathbf{1}$ & $6.9 \%$ & $8.1 \%$ & $8.7 \%$ & $9.0 \%$ & $9.1 \%$ & $9.2 \%$ & $9.3 \%$ \\
\cline { 2 - 9 } & $\boldsymbol{N}=\mathbf{2}$ & $6.3 \%$ & $9.0 \%$ & $10.3 \%$ & $11.0 \%$ & $11.3 \%$ & $11.5 \%$ & $11.6 \%$ \\
\hline
\end{tabular}


using $N=2$ has higher demands on the switches on-resistance that a system using only $N=1$.

\section{Charging The Supercapacitor MOdUle}

For the purpose of charging supercapacitors there are some charging ICs available on the market, with subtle differences from manufacturer to manufacturer. Notwithstanding, a typical supercapacitor charger IC can operate from a DC voltage supply and charge the supercapacitor at a constant current until the output voltage reaches a programmable value, thus preventing overcharging and physical disruption of the device.

In the presented stacking scheme the cells are not always in the same topology, hence the charging cannot be made by simply connecting a charger to the whole supercapacitor module. In which case, as the charging of supercapacitors should be fast, the switches would have to withstand large currents, leading to undesirable ohmic losses due to their on-resistance. To overcome these problems, we propose a parallel charging scheme, as illustrated in Fig. $3^{2)}$.

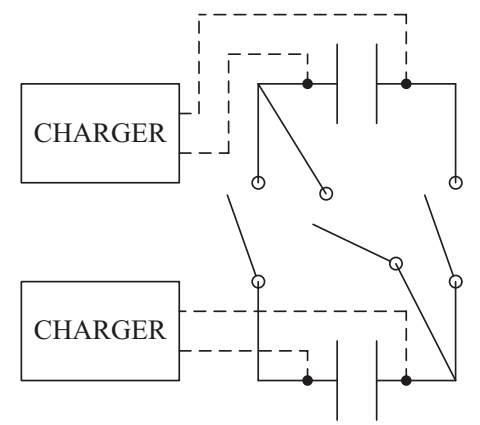

Figure 3. Supercapacitor module charging scheme (for $N=1$ )

In this scheme, one charger connects to each supercapacitor cell, so that the charging of all the cells can be done in parallel. If the chargers are identical and charging at the same current, there would be no charging current flowing through the switches. However, a typical supercapacitor charger IC doesn't have a differential output, as this would be needed to use the presented charging scheme to charge the whole module from a single DC voltage source. This way, each charger needs to operate with a different floating ground, which can be achieved by connecting a different voltage source to each charger.

Fig. 4 shows simulation results when this charging scheme is applied to a two supercapacitor stacking scheme $(N=1)$, using Linear Technology's LTC3225 as the supercapacitor charger. The maximum output current of this charger is only $150 \mathrm{~mA}$, but it perfectly illustrates this charging scheme. This simulation depicts the situation when the mobile phone is in standby and the charging is started after the first stacking operation is made, therefore when the two cells are placed in series connection. Then, when the bottom cell voltage $V_{\mathrm{M}}$ reaches $V_{\mathrm{S}}$, the stacking scheme does the opposite of what it does when the supercapacitors are discharging, this time, placing them in parallel connection (lowering the total module voltage). This is summarized in Fig. 4, where the Chargers row indicates whether the chargers are $\mathrm{ON}$ or $\mathrm{OFF}$ and the Topology row shows if the supercapacitors are in series $(\mathrm{S})$ or in parallel $(\mathrm{P})$ connection. With this scheme, even when the

2) The presented charging scheme is designed for a supercapacitor module with $N=1$ for simplicity in understanding the approach to the problem of charging the whole module, as this principle is independent of $N$

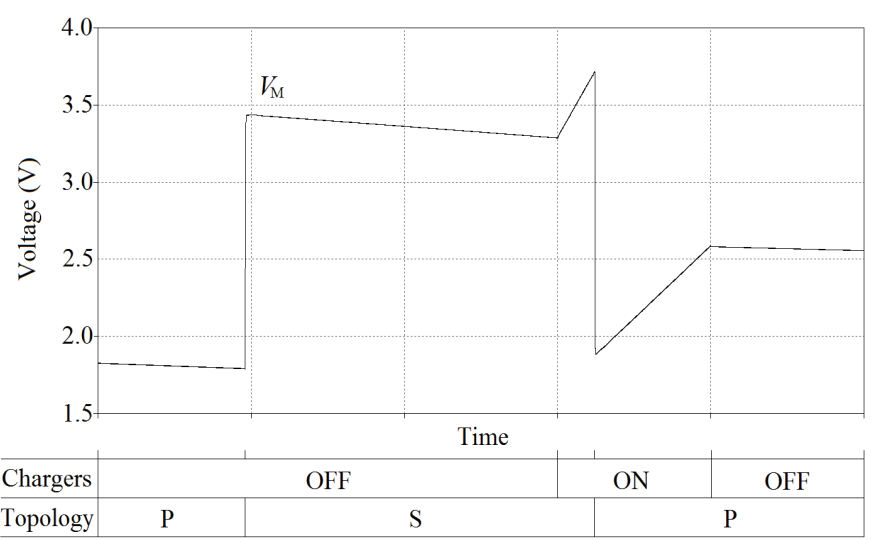

Figure 4. Supercapacitor module voltage behavior during the charging $(N=1)$

mobile phone is being charged, the input voltage for the boost converter is always in its higher efficiency range, thus allowing incremental charges.

\section{CONCLUSIONS}

The presented circuit allows a mobile phone to operate efficiently using supercapacitors as its energy source, with all the benefits that this technology may provide, such as fast charging cycles and low energy incremental charging. This is achieved through the combined use of a boost converter and a voltage controlled supercapacitor switching module. This scheme can improve the performance of general-purpose step-up converters and augment the amount of total usable stored energy. As the simulation results show, the gain in the autonomy of the device depends on the step-up converter itself, and on parameters such as the minimum operating input voltage, or the converter's efficiency for different ranges of input voltage, allowing higher autonomy gains to be obtained with an application specific step-up converter design.

This design is also charging compatible, as it unstacks the supercapacitors, allowing each one of the cells in the module to be charged independently, thus allowing faster charging.

As future work, we will be prototyping this design and use this technology in applications requiring fast charging and/or low energy incremental charging, such as using human kinetic energy harvesting for charging mobile devices, without compromising the presented scheme's operability.

\section{REFERENCES}

[1] A. Burke, "Ultracapacitors: why, how, and where is the technology", Journal of Power Sources 91, pp. 37-50, 2000

[2] A.Schneuwly, R. Gallay, "Properties and applications of supercapacitors: from the state-of-the-art to future trends", Proceedings of PCIM, 2000

[3] J. Schindall, "What's in a name? A new model for regenerative electrical energy storage", Power Electronics Society Newsletter, vol. 20, number 1, First Quarter 2008

[4] R. Signorelli, D. Ku, J. Kassakian, J. Schindall, "Electrochemical double-layer capacitors using carbon nanotube electrode structures", Proceedings of the IEEE, vol. 97, No. 11, November 2009

[5] P. Barrade, S. Pittet, A. Rufer, "Series connection of supercapacitors, with an active device for equalizing the voltages", Proceedings of PCIM, 1999 